\title{
Correction to: An Efficient Retrieval Method for Astronomical Catalog Time Series Data
}

\author{
Bingyao Li, Ce Yu, Xiaoteng Hu, Jian Xiao, Shanjiang Tang, \\ Lianmeng $\mathrm{Li}$, and Bin Ma
}

\section{Correction to: \\ Chapter "An Efficient Retrieval Method for Astronomical Catalog Time Series Data" in: J. Vaidya and J. Li (Eds.): Algorithms and Architectures for Parallel Processing, LNCS 11334, https://doi.org/10.1007/978-3-030-05051-1_20}

The original version of the chapter starting on p. 284 was revised. The grant numbers of the Joint Research Fund in Astronomy were incorrect in the acknowledgement on p. 297. The original chapter was corrected. 